\title{
Global Semantic Graph as an Alternative Information and Collaboration Infrastructure
}

\author{
Yan Shvartzshnaider \\ School of Electrical and Information Engineering, \\ The University of Sydney, Australia \\ yshv6985@uni.sydney.edu.au \\ www.ee.usyd.edu.au
}

\begin{abstract}
We propose the development of a Global Semantic Graph (GSG) as the foundation for future information and collaboration-centric applications and services. It would provide a single abstraction for storing, processing and communicating information based on globally interlinked semantic resources. The GSG adopts approaches and methods from the Semantic Web and thus facilitates a better information sharing abstraction.
\end{abstract}

\section{Problem and Motivation}

New and emerging Web technologies are gradually transforming the way information is communicated. In particular, popular social networks sites such as Facebook, MySpace and Twitter represent a real shift in the communication paradigm. The latest paradigm puts the focus on information itself and draws on collaboration methods between users, applications and services. The core building block for collaboration between applications is sharing/syncing of information or state, however, now it is not just about syncing within a specific application or context, but rather into a single global context. A prime example of such is the recently unveiled Google Wave [1, where users collaborate within a shared context of a single document (i.e., Wave). Despite their enhanced capabilities, however, Google Wave and other upcoming technologies are strictly limited to social interactions and operate in isolation from other existing Web applications.

Therefore, as the Internet's usage patterns gradually become more involved with multiple interactions between a variety of services and systems, there is an increasing demand for an architecture and infrastructure for interconnectivity and information exchange between applications on a global level. Although some applications may interoperate, for example, an email client might have an access to a contacts manager 2, the current Internet architecture does not provide the desired interoperability [3] as "there is no consistent approach for allowing interoperation and a system wide exchange of data between applications 2]." So, each application operates on a separate data structure and is unaware of related and relevant data in other applications $[2$.

L. Aroyo et al. (Eds.): ESWC 2010, Part II, LNCS 6089, pp. 483-487, 2010.

(C) Springer-Verlag Berlin Heidelberg 2010 
Motivated by these shortcomings of the Internet architecture and inspired by the new collaboration approaches, we propose a standardised architecture for information capture as well as communication between different information realms in the form of a global semantic graph (GSG). The GSG is a persistent, globally shared information space that is securely accessible and manipulated from/by any application and service.

In other words, the GSG forms a single global context within which the applications sync their respective data and state, so that it can be accessed and used for a variety of collaboration and interoperability purposes.

\section{Approach and Methodology}

In our approach, as depicted by Figure 1, we propose to have all future collaboration and communication processes carried out via the GSG. The GSG adopts approaches and methods from the Semantic Web that provides the "means to build the semantic bridges necessary for data exchange and application integration [2]" It is essentially, large-scale distributed tuple store to which applications and services simply "publish" their internal state changes via simple tuple insertion while a "subscription" is essentially a standing query that keeps the internal state synchronised with any new information provided by others.

We are currently interested in the following research questions:

1. How to insure good load balancing, scalability and information routing capabilities in a global storage system?

2. What is most efficient way to implement the pub/sub paradigm on top of a scalable semantic graph?

3. What is a proper security model that will work well with the GSG?
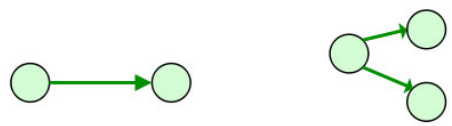

Mapping from global graph via patternSpec

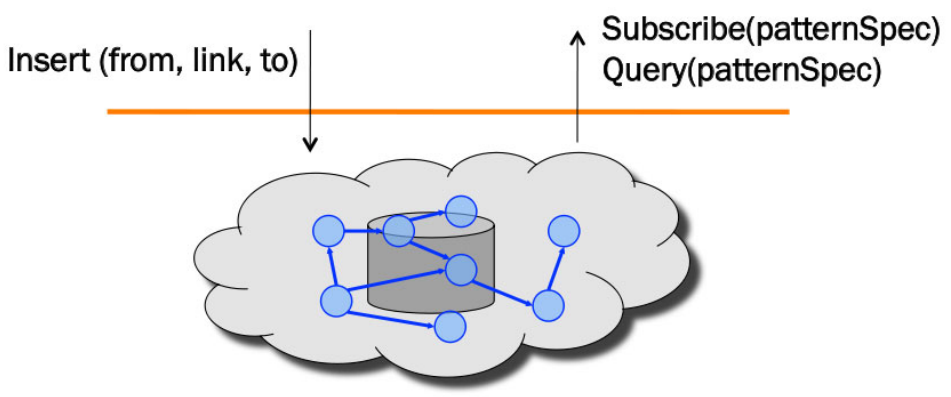

Fig. 1. The GSG combines Network, Database and Semantic Web in a single abstraction. Information is "filtered" by the subscription to a particular context pattern (e.g. John's emails) and then mapped on to a specific application (e.g. message reader). 
To answers the above questions we are examining the work in the following research areas:

Large tuple store. We are considering the utilisation of a DHT-based Structured P2P overlays distributed storage system. However, while, on one hand it provides efficient data item discovery and uniform load distribution, in addition to self-organisation and node failure tolerance, on the other hand the DHT inherently does not support much needed wildcard and multi-attribute queries.

Publish/Subscribe communication paradigm. Our approach builds on evaluation of long-standing queries, also called continuous queries, which are issued once and remain in the system to return results every time the database is updated. We are looking at porting this approach to a large-scale distributed tuple store where an active standing query evaluation would act as a filter on the store's data and effectively provide a pub/sub service. In the case of the GSG, a standing query is essentially a set of tuple templates (or conditions) that return matching tuples. Therefore, an implementation of an efficient pattern matching algorithm (e.g. RETE [4]) with data-dependent, dynamic template re-writing on top of a distributed environment might serve as a possible solution.

Reasoning. The GSG architecture provides support to "anytime" distributed reasoning, similarly to the MARVIN [5] platform. Reasoning agents compute the deductive closure of the subscribed parts of the graph, and publish the results back into the GSG.

Security. We have some thoughts that involve using Named Graphs [6] for authentication and authorisation controls, however these initial ideas require further research and discussions with security experts.

To address above challenges we plan to follow the following research methodology: first, we will conduct an extensive literature review of current state-ofthe-art technologies and methods in order to better understand them. Second, we plan to implement an efficient pattern matching algorithm and integrate it into a prototype system. Next, we will deploy the prototype system on top of a distributed environment through a testbeds framework (for LAN and WAN). Finally, we seek to evaluate the results and in order to optimise the system for better overall performance.

\section{Results}

This $\mathrm{PhD}$ research is a continuation of an Engineering Honours project thesis which looked into the GSG as an alternative to the Internet's information communication infrastructure. In particular, we were interested to see how the GSG abstraction impacts on the design and implementation of new services. That research project was awarded "The Best Research Project" prize in the Engineering Sydney Research Conversazione 20091, organised by The University

\footnotetext{
${ }^{1}$ http://www.eng.usyd.edu.au/engineeringsydney/conversazione.shtml
} 
of Sydney. We are currently in the process of implementing and subsequently testing the GSG prototype. In particular, we have developed a basic prototype of a pub/sub system based on the RETE [4] algorithm. At the time of writing, the prototype was successfully deployed on top of the OMF (cOntrol and Management Framework $\sqrt[2]{2}$ testbed.

\section{Related Work}

The implementation of the GSG builds on many existing standards and systems. Due to space restrictions, we only discuss here related work highlights, in two key research areas: globally distributed content storage and the publish-subscribe communication paradigm.

Distributed storage: The increasing demand for online services has prompted a number of research efforts both in the academic community and the industry to develop a robust and scalable storage systems.

For example, Amazon's Dynamo [7] is a highly available key-value global storage system. Dynamo is guided by Amazon's e-commerce target requirements, which are mainly concerned with providing an "always-on" experience, even at a cost of temporarily sacrificing information consistency. Consequently, Dynamo completely lacks any security or data integrity mechanisms as it was designed to operate in a fully trusted domain. Nevertheless, there are a number of Dynamo's design aspects and assumptions that we see useful in relation to the GSG implementation. These include the use of a Distributed Hash Table (DHT) for data storage and routing, the "always-writeable" data store assumption (i.e. no updates, only writes), failures handling, versioning and replica synchronisation protocols.

In other related work, RDFPeers [8], one of the first distributed RDF stores was developed on top of Multi-Attribute Addressable Network (MAAN) 9]. RDFPeers supports atomic, disjunctive and conjunctive multi-predicate RDF queries, however it suffers from poor load balancing capabilities [10].

Publish/Subscribe communication paradigm: The implementation of a pub/sub schema on a global, Internet like scale is still an open research problem. Interesting work is being done by the EU funded PSIRP ${ }^{3}$ project 11 that aims to develop an information-centric internetworking architecture based on the publish-subscribe paradigm 12 . The PSIRP project employs label switching forwarding to deliver information [3] and in this it differs to our approach described in Section 1 .

\section{Conclusion and Future Work}

We have described our vision and motivation for a new Internet informationcentric collaboration architecture in a form of a global semantic graph (GSG).

\footnotetext{
${ }^{2}$ http://omf.mytestbed.net/

${ }^{3}$ Publish-Subscribe Internet Routing Paradigm
} 
GSG's design is motivated by the poor ability of Internet's architecture to provide the desired interoperability between applications 3 . The GSG is a persistent, distributed global graph that provides storage as well as publish/subscribe based communication. It differs from classical pub-sub systems in that publications to it "permanently" persist, and that the subscriptions are on the shared graph and not on the individual publications.

Our approach builds on many existing standards and systems that need to work together. Therefore, the implementation aspects include many challenges in a wide range of research areas. In particular, we wish to find a way to facilitate multi-attribute and range queries over distributed large-scale storage systems without compromising scalability and load balancing properties. Moreover, implementing a pub/sub communication paradigm on a global scale based on a persistent shared-state, together with the security aspects, is a clear must for a global system.

To seek answers to these and other related research questions, we plan to follow the methodology set out in Section 1, to develop a prototype implementation for the GSG.

\section{References}

1. About Google Wave (January 2010), http://wave.google.com/help/wave/about.html

2. Groza, T., et al.: The Nepomuk Project - On the way to the Social Semantic Desktop. In: Proc. of I-Semantics, vol. 7, pp. 201-211 (2007)

3. Sarela, M., Rinta-aho, T., Tarkoma, S.: RTFM: Publish/subscribe Internetworking Architecture. In: ICT Mobile Summit, Stockholm (2008)

4. Forgy, C.: A Network Match Routine for Production Systems. Working Paper, Tech. Rep. (1974)

5. Anadiotis, G., et al.: MaRVIN: a distributed platform for massive RDF inference (2008), http://www. larkc.eu/marvin/btc2008.pdf

6. Carroll, J., Bizer, C., Hayes, P., Stickler, P.: Named Graphs, Provenance and Trust. In: Proc. of the 14th Int. Conf. on the World Wide Web, p. 622 (2005)

7. DeCandia, G., et al.: Dynamo: Amazon's Highly Available Key-value Store. ACM SIGOPS Operating Systems Review 41(6), 220 (2007)

8. Cai, M., Frank, M.: RDFPeers: a scalable distributed RDF repository based on a structured peer-to-peer network. In: Proc. of the 13th Int. Conf. on the World Wide Web, p. 657 (2004)

9. Cai, M., et al.: MAAN: A multi-attribute addressable network for grid information services. Journal of Grid Computing 2(1), 3-14 (2004)

10. Anadiotis, G., Kotoulas, S., Siebes, R.: An Architecture for Peer-to-Peer Reasoning. In: Proc. of 1st Workshop on New forms of reasoning for the Semantic Web, International Semantic Web Conference, ISWC 2007, Busan, Korea (2007)

11. Fotiou, N., Polyzos, G., Trossen, D.: Illustrating a Publish-Subscribe Internet Architecture. In: 2nd Euro-NF Workshop (2009)

12. PSIRP project homepage (December 2009), http://www.psirp.org/ 